\title{
Fortalezas Humanas y Bienestar Biopsicosocial: Revisión sistemática
}

\section{Character Strengths and Biopsychosocial Well-being: a systematic review}

\author{
María Mercedes Ovejero Bruna ${ }^{1}$, Violeta Cardenal Hernáez ${ }^{1}$, Margarita Ortiz-Tallo ${ }^{2}$ \\ ${ }^{1}$ Universidad Complutense de Madrid (España). ${ }^{2}$ Universidad de Málaga (España)
}

Disponible online 31 de diciembre de 2016

\begin{abstract}
Este trabajo presenta una revisión sistemática sobre las fortalezas psicológicas propuestas por el modelo Values in Action (VIA) y su relación con el bienestar, la satisfacción vital, la felicidad, la resiliencia y la salud en diferentes poblaciones. Los resultados de la revisión de 47 estudios permiten concluir que las fortalezas psicológicas están relacionadas positivamente con la satisfacción vital, el bienestar, la felicidad, la resiliencia y la salud, y en menor medida con indicadores de afecto negativo. Estos resultados son similares en distintos contextos y en diversos grupos de edad. Se discuten los hallazgos de los diversos estudios y se proponen implicaciones y perspectivas futuras del estudio y del desarrollo de las fortalezas psicológicas en los individuos, en los grupos y en la sociedad en general.

Palabras clave: Fortalezas psicológicas; Modelo VIA; Resiliencia; Bienestar; Felicidad; Salud

This study is a systematically review of the Values in Action (VIA) model of virtues and strengths and its correlation with well-being, life satisfaction, happiness, resilience, and health in different populations. A total of 47 studies were reviewed. The results showed that psychological strengths were associated with higher levels of happiness, wellbeing, resilience, health, and positive affect, and weakly associated with negative affect. These results are similar across different settings and ages. We discuss the results of the studies and consider their implications for future research related to the development of psychological strengths in individuals, groups, and society.
\end{abstract}

Keywords: Character strengths, VIA Model; Resilience; Well-being; Happiness; Health

Correspondencia: María Mercedes Ovejero Bruna. Departamento de Personalidad, Evaluación y Tratamiento psiclógico. Facultad de Psicología. Campus de Somosaguas. 28223 POZUELO DE ALARCÓN (MADRID). E-mail: mercheovejero@psi.ucm.es. E-mail de las coautoras: Violeta Cardenal Hernáez, vcardenal@psi.ucm.es; Margarita Ortiz-Tallo, mortiztallo@uma.es 
La Psicología ha adolecido de un vocabulario común y una aproximación a la métrica de los rasgos positivos antes del año 2004. El enfoque tradicional de la Psicología se ha beneficiado de sistemas como la Clasificación Diagnóstica de los Trastornos Mentales (DSM, American Psychiatric Association, 2013) ya que proveía a los investigadores, académicos y clínicos de un lenguaje común para operativizar la Psicopatología. Como primer paso para solventar la disparidad entre este enfoque más clásico y el basado en la Psicología Positiva, Peterson y Seligman (2004) establecieron como objetivo la identificación y medida de lo que en un inició se denominó el "buen carácter". Desde este planteamiento surgió el modelo Values in Action (VIA), el cual presenta un marco de trabajo basado en el potencial de las personas que es ampliamente aplicado para ayudar a descubrir, explorar y utilizar aquellas cualidades que son positivas (Niemiec, 2013). El origen de esta clasificación se sitúa en la revisión de diferentes textos históricos, filosóficos, religiosos, políticos, virtudes de figuras históricas, de fuentes imaginarias (por ejemplo, personajes de la literatura, del cine y del arte) y consultas a expertos en la temática llegándose a elaborar un listado de virtudes y fortalezas cuyo objetivo es fomentar el bienestar y el éxito individual y grupal (Dahlsgaard, Peterson y Seligman, 2005).

Las virtudes se definen como aquellos rasgos centrales del carácter valorados moralmente que han estado y están presentes universalmente, tienen sus raíces en la biología y han sido evolutivamente seleccionadas (Noftle, Schnitker y Robins, 2011; Peterson y Park, 2009). Peterson y Seligman (2004) organizaron cada una de estas virtudes de manera más concreta, surgiendo así las fortalezas. Éstas se definen como características psicológicas que se presentan en distintas situaciones y a lo largo del tiempo y a las que se les otorga un valor moral (Peterson y Park, 2009). Son los ingredientes psicológicos de las virtudes y menos abstractas que éstas (Noftle et al., 2011; Peterson, 2006a), ya que se manifiestan en los pensamientos, emociones y conductas de la persona (Niemiec, 2010) y permiten a las personas dar lo mejor de sí mismas (Wood, Linley, Maltby, Kashdan y Hurling, 2011). Según Peterson y Seligman (2004), hay 24 fortalezas (ver Tabla 1).

Para la evaluación de las fortalezas desde el modelo VIA, se han creado varios instrumentos de evaluación, siendo los más utilizados el VIA-IS (Peterson, Park y Seligman, 2005) para la evaluación de las 24 fortalezas en adultos y el VIA-Y (Park y Peterson, 2003a; 2003b), diseñado para evaluar las fortalezas en niños y adolescentes entre 10 a 17 años. Estos instrumentos han sido objeto de complejos estudios psicométricos (McGrath 2014; 2015), y además han sido aplicados para estudiar las diferencias entre hombres y mujeres (véase Ovejero y Cardenal, 2015 para una revisión de estudios sobre esta temática), explorar la conexión entre las fortalezas y los rasgos de personalidad (Macdonald et al. 2008; Noftle et al., 2011), y especialmente para estudiar la conexión entre las fortalezas y el bienestar en diferentes contextos, siendo los primeros estudios los publicados por Park, Peterson y Seligman (2004a; 2004b).
Tabla 1

Las fortalezas humanas.

Sabiduría y conocimiento

Curiosidad: Interés por explorar el mundo, encontrar temas fascinantes y descubrir nuevas cosas.

Amor por el conocimiento: Deseo por adquirir nuevas habilidades o aprendizajes, espontánea o formalmente.

Pensamiento crítico: Pensar sobre las cosas y examinar todos sus significados y matices. Estar dispuesto a cambiar las propias ideas según la evidencia.

Creatividad: Capacidad para producir nuevas y productivas formas de conceptualizar y hacer las cosas.

Perspectiva: Ser capaz de dar consejos sabios y adecuados a los demás, encontrando caminos no solo para comprender el mundo, sino para facilitar su comprensión a las demás personas.

\section{Coraje}

Valentía: Defender una postura aunque exista oposición por parte de los demás, actuar según las propias convicciones aunque suponga ser criticado.

Persistencia: Persistir en algo aunque existan obstáculos. Obtener satisfacción por las tareas emprendidas y que consiguen finalizarse con éxito.

Integridad: Vivir la vida de forma genuina y auténtica, ser sincero y responsable de los propios sentimientos y acciones.

Vitalidad: Afrontar la vida con entusiasmo y energía, incluso en situaciones difíciles.

\section{Humanidad}

Generosidad: Hacer favores y buenas acciones, ayudando a otras personas y disfrutando con ello.

Amar y dejarse amar: Tener importantes y valiosas relaciones con otras personas, en particular con aquellas en las que el afecto y el cuidado son mutuos.

Inteligencia emocional, personal y social: Ser consciente de las emociones y sentimientos de uno mismo y de los demás.

\section{Justicia}

Trabajo en equipo: Trabajar bien en equipo, ser fiel al grupo y sentirse parte de él. Incluye el sentimiento de pertenecer a una comunidad.

Imparcialidad: Tratar a todas las personas como iguales con equidad y justicia.

Liderazgo: Animar al grupo del que uno es miembro para hacer cosas, así como reforzar las relaciones entre las personas de dicho grupo. Organizar actividades grupales y llevarlas a buen término.

\section{Templanza}

Autorregulación: Capacidad para gestionar los propios sentimientos y acciones. Tener disciplina y control sobre los impulsos y emociones.

Prudencia: Ser cauteloso para tomar decisiones, no asumiendo riesgos innecesarios. Pensar en los beneficios a largo plazo de una acción.

Modestia: Dejar que sean los demás los que hablen por sí mismos, no buscar ser el centro de atención y no creerse más especial que los demás.

Perdón: Perdonar a aquellas personas que han actuado mal, no mostrando sentimientos de venganza.

$$
\text { Trascendencia }
$$

Apreciación de la belleza: Saber apreciar la belleza de las cosas o interesarse por aspectos de la vida como la naturaleza, el arte, la ciencia, etc.

Gratitud: Ser consciente y agradecer las cosas buenas que suceden. Expresar agradecimiento.

Esperanza: Esperar lo mejor para el futuro y trabajar para conseguirlo. Creer que un buen futuro es algo que está en nuestras manos conseguir.

Espiritualidad: Pensar que existe un propósito o significado universal en las cosas que ocurren en el mundo y en la propia existencia. Tener creencias coherentes sobre un propósito más alto y un sentido en la vida.

Humor: Gustar de reír y gastar bromas, sonreír con frecuencia, ver el lado positivo de la vida. Hacer reír a otras personas.

Nota. Adaptado de Peterson y Seligman (2004) 
El objetivo del presente trabajo es establecer el estado de la cuestión con respecto a la conexión entre las fortalezas y el bienestar, la felicidad, la satisfacción vital, la resiliencia y la salud mental. Para ello se ofrece una revisión teórica de estudios basados en el modelo VIA que analicen la relación con estas variables en diferentes poblaciones procedentes de diversos países. Mediante este objetivo se pretende aunar la evidencia empírica actual y hacer balance de resultados para analizar las implicaciones de los resultados.

\section{Revisión sistemática}

\section{Método}

La búsqueda de información se llevó a cabo por parte de todas las autoras del presente trabajo y de manera independiente, almacenándose las referencias en un archivo digital compartido a través de Google Docs. En todo momento, se guardó el máximo parecido con el procedimiento empleado en las revisiones del sistema PRISMA. Se emplearon las bases de datos PsychINFO, PsicARTICLES, Psicodoc, PubMed, Medline y Psychological Abstracts empleando como rango de fechas los años entre 2004 y 2016 ambos incluidos. Como suplemento se emplearon Google Scholar, Cross Ref y Research Gate, siendo utilizado este último recurso para localizar investigadores especialistas en la temática objeto de estudio y así solicitar los textos completos en caso de no disponibilidad de los mismos en las fuentes primarias de consulta. Las palabras clave empleadas se dividieron en 3 grupos:

Grupo 1: Character strengths; Values in Action; VIA; Values in Action for Youth; VIA-Y; Fortalezas del carácter; Fortalezas.

Grupo 2: Well-being, Bienestar; Happiness; Felicidad; Afecto positivo; Positive affect; Afecto negativo; Negative Affect; Salud; Health; Resiliencia; Resilience.

Grupo 3: Niños; Children; Adolescentes; Adolescents; Teenagers; Ancianos; Personas mayores; Elderly.

En una primera fase se emplearon palabras clave del grupo $1 \mathrm{y}$ del grupo 2. Una vez finalizada esta primera secuencia, se procedió a aplicar palabras clave del grupo 1 y del grupo $2 \mathrm{y}$ del grupo 3. Por otra parte, para la selección final de los artículos a introducir en la base de datos, se siguieron los criterios de inclusión y exclusión especificados en la Tabla 2 (siguiendo los estándares PRISMA, Institute of Medicine of the National Academies, 2011).

Tras la lectura y revisión de los resúmenes, y aplicando estos criterios de inclusión y exclusión, se seleccionó finalmente un total de 47 trabajos. El flujo completo de artículos de la búsqueda empleando el sistema PRISMA de reporte gráfico (Moher, Liberati, Altman y The PRISMA Group, 2009) se especifica en la Figura 1.
Tabla 2

Criterios de inclusión y exclusión.

\begin{tabular}{|c|c|}
\hline Inclusión & Exclusión \\
\hline $\begin{array}{l}\text { Fechas: } 2004-2016 \text {. } \\
\text { Artículos que empleen el instrumento } \\
\text { VIA-IS o el VIA-Y. } \\
\text { Artículos que evalúen la satisfacción } \\
\text { vital, el bienestar, la felicidad, el } \\
\text { afecto positivo/negativo, la salud y la } \\
\text { resiliencia de manera cuantitativa. } \\
\text { Artículos escritos en inglés, español, } \\
\text { francés. }\end{array}$ & $\begin{array}{l}\text { Artículos fuera del rango de fecha } \\
\text { establecido. } \\
\text { Aplicación de otro enfoque de } \\
\text { fortalezas diferente del VIA. } \\
\text { Aplicación de otro instrumento } \\
\text { diferente del VIA-IS o del VIA-Y. } \\
\text { Publicaciones en otros idiomas } \\
\text { diferentes del inglés, español, francés. } \\
\text { Aproximaciones cualitativas. } \\
\text { Texto completo no disponible. } \\
\text { Artículos en prensa o en fase de } \\
\text { revisión. }\end{array}$ \\
\hline
\end{tabular}

Figura 1

Flujo de estudios primarios. (Adaptado de Moher, Liberati, Altman y The PRISMA Group, 2009)
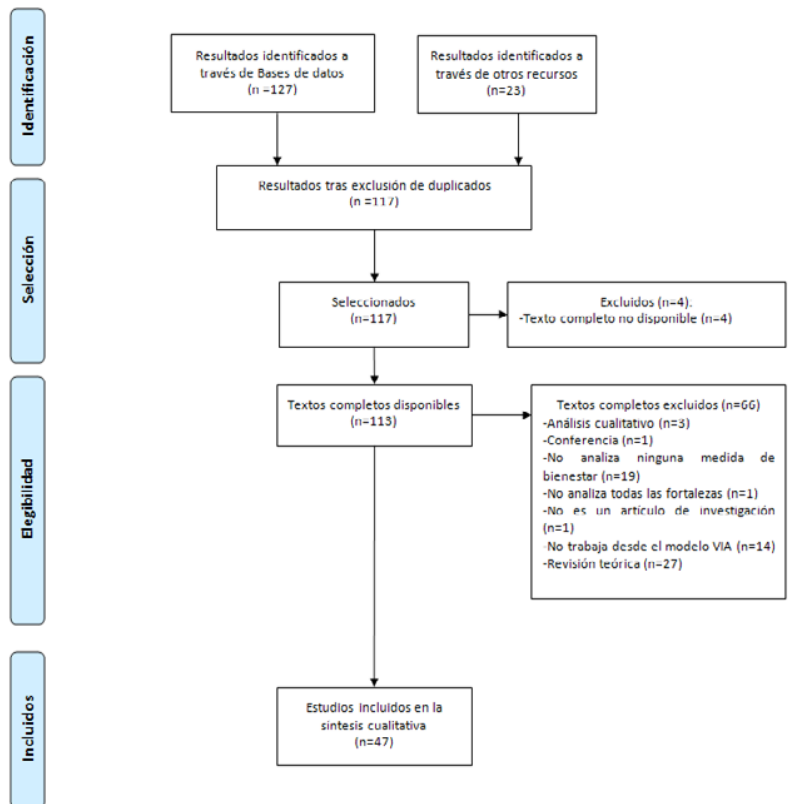

Una vez elaborado el listado de artículos candidatos a la inclusión en la revisión sistemática, la codificación se hizo por dos de las autoras del presente trabajo. Ésta se hizo de manera independiente en un archivo Excel con la misma estructura y libro de códigos para ambas codificadoras. Posteriormente, una tercera evaluadora detectó las discrepancias entre ambas bases de datos. Una vez terminada su detección, se programó una teleconferencia en la que se resolvieron las diferencias en codificación. Se obtuvo un consenso absoluto para todos los artículos seleccionados. La Tabla 3 presenta un resumen de características de los artículos primarios que componen la revisión. 
FORTALEZAS HUMANAS Y BIENESTAR BIOPSICOSOCIAL: REVISIÓN SISTEMÁTICA

Tabla 3

Artículos seleccionados para la síntesis cualitativa.

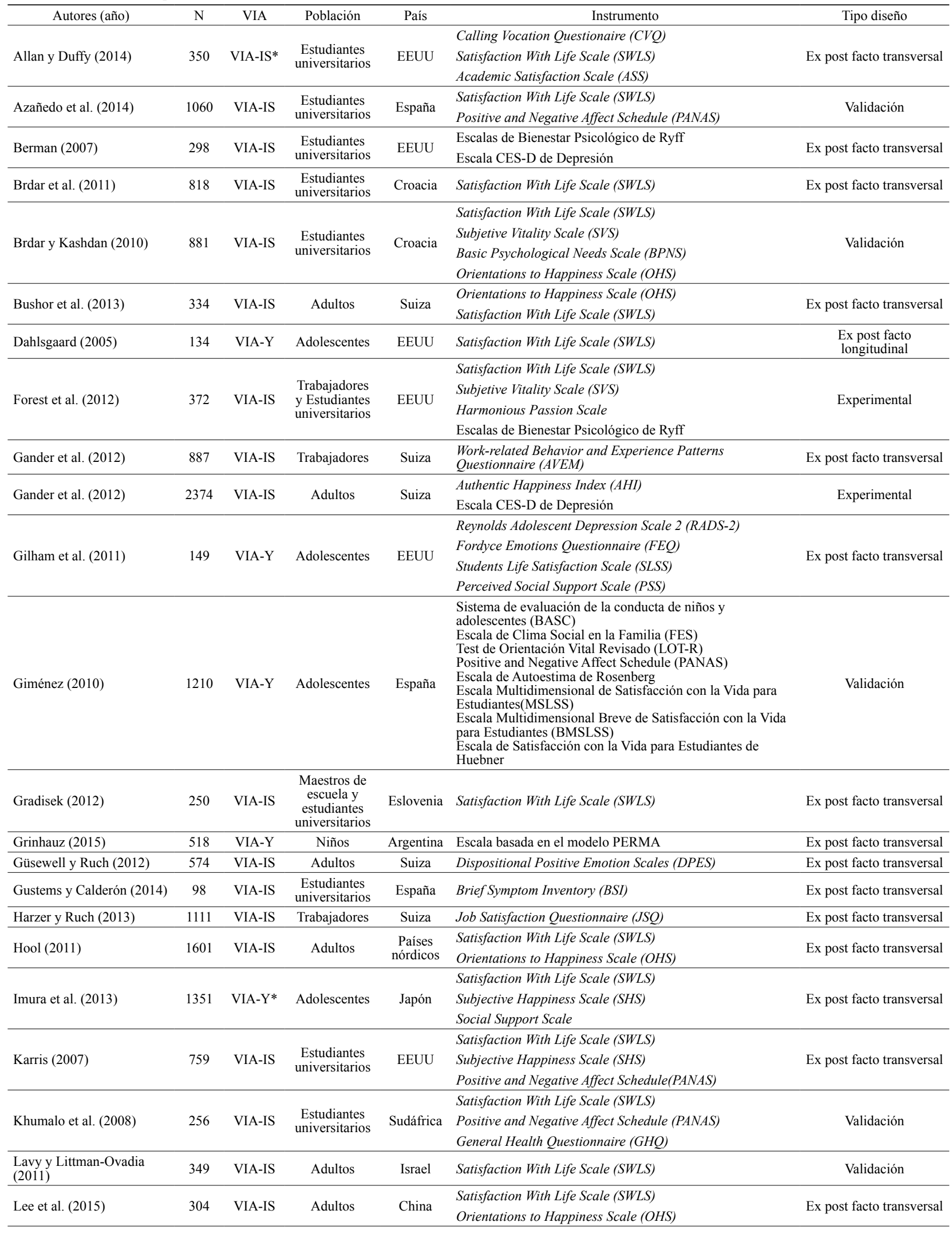




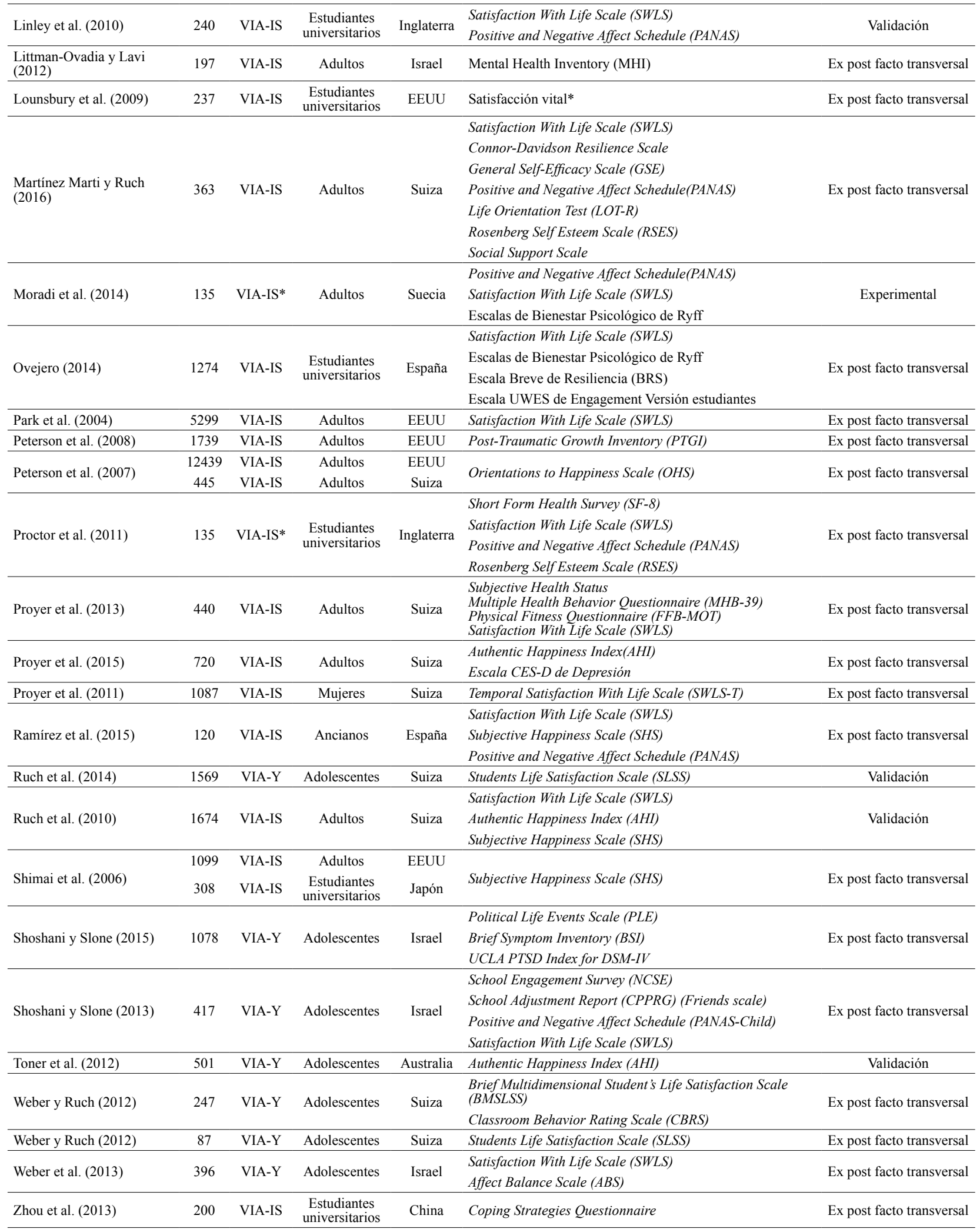

Nota. *Estos estudios emplean una variante del VIA específica. 


\section{Resultados}

Los resultados de la revisión sistemática, organizados por variables y fortalezas se ofrecen en la Tabla 4.

Tabla 4

Resumen de los resultados de la revisión sistemática.

\begin{tabular}{|c|c|c|c|c|c|c|}
\hline Fortaleza & Satisfacción vital & $\begin{array}{c}\text { Bienestar } \\
\text { psicológico/Felicidad }\end{array}$ & Afecto Positivo & Afecto negativo & Salud física/mental & $\begin{array}{c}\text { Resiliencia / Estilos } \\
\text { de afrontamiento }\end{array}$ \\
\hline Creatividad & $*$ & $* * *$ & $* *$ & NS & NS & $*$ \\
\hline Curiosidad & $* * *$ & $* * *$ & $* *$ & $(-)^{*}$ & $(-)^{*}$ & $* *$ \\
\hline Juicio crítico & $*$ & $*$ & $* *$ & $(-)^{*}$ & $(-)^{*}$ & $*$ \\
\hline $\begin{array}{l}\text { Amor por el } \\
\text { conocimiento }\end{array}$ & $*$ & $* * *$ & $* *$ & NS & $(-)^{*}$ & $*$ \\
\hline Perspectiva & $* *$ & $* * *$ & $* *$ & $(-)^{*}$ & $(-)^{*}$ & $* *$ \\
\hline Valentía & $*$ & $* * *$ & * & $(-)^{*}$ & $(-)^{*}$ & $* *$ \\
\hline Persistencia & $* *$ & $* *$ & * & NS & $(-)^{*}$ & $*$ \\
\hline Honestidad & $*$ & $* *$ & $*$ & $(-)^{*}$ & $(-)^{*}$ & $*$ \\
\hline Vitalidad & $* * *$ & $* * *$ & $* *$ & $(-)^{*}$ & $(-)^{*}$ & $*$ \\
\hline Amor & $* * *$ & $* *$ & $*$ & $(-)^{*}$ & $(-)^{*}$ & NS \\
\hline Amabilidad & $*$ & $* *$ & $*$ & NS & NS & NS \\
\hline Inteligencia Social & $*$ & $* *$ & $* *$ & $(-)^{*}$ & $(-)^{*}$ & $*$ \\
\hline Civismo & $*$ & $*$ & * & $(-)^{*}$ & NS & NS \\
\hline Imparcialidad & $*$ & $* *$ & $*$ & NS & NS & NS \\
\hline Liderazgo & $*$ & $* *$ & $*$ & NS & $(-)^{*}$ & $*$ \\
\hline Perdón & $*$ & $* *$ & $*$ & $(-)^{*}$ & NS & $*$ \\
\hline Modestia & NS & $*$ & NS & NS & NS & $*$ \\
\hline Prudencia & NS & $*$ & $*$ & NS & NS & NS \\
\hline Auto-regulación & NS & $*$ & $* *$ & $(-)^{*}$ & $(-)^{*}$ & $*$ \\
\hline $\begin{array}{l}\text { Apreciación de la } \\
\text { belleza }\end{array}$ & NS & $* *$ & $*$ & NS & NS & NS \\
\hline Gratitud & $* * *$ & $* * *$ & $* *$ & $(-)^{*}$ & $(-)^{*}$ & $*$ \\
\hline Esperanza & $* * *$ & $* * *$ & $* *$ & $(-)^{*}$ & $(-)^{*}$ & $* * *$ \\
\hline Humor & $* *$ & $*$ & $*$ & $(-)^{*}$ & $(-)^{*}$ & $* *$ \\
\hline Espiritualidad & $*$ & $* * *$ & $*$ & NS & NS & NS \\
\hline
\end{tabular}

Nota. $* * *$ Correlación superior a $.40 . * *$ Correlación entre .30 a .39. * Correlación entre .10 a .29 . NS: Correlación no significativa o próxima a 0. (-): Correlación negativa o inversa.

En general, los resultados de la revisión cualitativa muestran que las fortalezas están relacionadas especialmente con la satisfacción vital, el bienestar, la felicidad, la resiliencia $\mathrm{y}$ en menor medida con indicadores de afecto negativo y de salud mental. Además se observa que hay fortalezas que sistemáticamente están relacionadas con mayores niveles de bienestar: curiosidad, vitalidad, amor, gratitud y esperanza. Otro bloque de fortalezas muestran escasa significación con los indicadores seleccionados para este trabajo: modestia, prudencia y auto-regulación. Estos resultados son similares en función del contexto y grupo de edad estudiado. Con respecto a la salud, se observa que una mayor puntuación en las fortalezas se relaciona con una menor tendencia a presentar problemas asociados a la depresión, ansiedad y estrés post-traumático entre otros.

\section{Discusión}

Desde sus inicios, el modelo VIA de fortalezas personales ha tenido como finalidad el análisis de aquellos rasgos que hacen que las personas den lo mejor de sí mismas y sean más felices.
Esto se operativiza a través de la medida de las fortalezas y de los diferentes aspectos del bienestar biopsicosocial.

En general, los resultados de la revisión sistemática muestran que la curiosidad, la vitalidad, el amor, la esperanza y la gratitud son importantes predictoras del bienestar, de la satisfacción vital y de la felicidad (Brdar, Anić y Rijavec, 2011; Gander, Proyer, Wyss y Ruch, 2012; Grinhauz, 2015; Güsewell y Ruch, 2012; Hool, 2011; Linley et al., 2010; Imura et al., 2013; Park, Peterson y Seligman, 2004a, 2004b; Peterson, Ruch, Beermann, Park y Seligman, 2007; Peterson, 2006; Proctor, 2013; Ramírez, Ortega y Martos, 2015; Wood, Joseph y Maltby, 2008; Wood et al., 2011), y además se relacionan con tener propósito en la vida (Bushor et al., 2013; Grinhauz, 2012; Peterson y Park, 2013).

La identificación y potenciación de fortalezas como la gratitud, la bondad, el trabajo en equipo y el humor se asocia con menores tasas de depresión (Gander et al., 2012; Gilham et al., 2011; Proyer et al.; 2015; Seligman, Steen, Park y Peterson, 2005), afecto negativo (Azañedo et al., 2014; Ramírez et al., 2015) y psicopatología (Shoshani y Slone, 2015), funcionando 
asimismo como amortiguador de los sucesos vitales de impacto negativo, convirtiéndose en importantes indicadores de un desarrollo saludable a largo plazo (Proyer, Gander, Wyss y Ruch 2011; Ruch, Proyer y Weber, 2010) pudiendo ayudar a construir la resiliencia (Martínez Marti y Ruch, 2016; Ovejero, 2014; Shoshani y Slone, 2015). Están relacionadas con la satisfacción y el bienestar laboral (Forest et al., 2012; Gander et al., 2012; Peterson, y Park, 2011; Peterson, Stephens, Park, Lee y Seligman, 2010) y el bienestar y la satisfacción en la escuela (Shoshani y Slone, 2012; Weber y Ruch, 2012).

Por otra parte, la modestia y la prudencia muestran una relación muy baja e incluso no relevante. Park et al. (2004a, 2004b) postularon que los resultados relacionados con la modestia pueden estar asociados al fomento del individualismo en las culturas occidentales. En lo que respecta a la prudencia y la auto-regulación, son fortalezas que actúan moderando la conducta y las emociones del individuo (Peterson y Seligman, 2004), por lo que los beneficios de estas fortalezas podrían estar presentes en otras áreas y variables no incluidas en el presente estudio.

En lo relativo a limitaciones, la más importante se relaciona con la inherente falta de una perspectiva cuantitativa que permita estudiar la intensidad de las correlaciones y el efecto de determinadas variables moderadoras, como puede ser la edad de los participantes de los estudios, la calidad métrica de los instrumentos empleados y/o la finalidad del estudio. El gran número de enfoques empleados para medir el bienestar en los estudios seleccionados implica que, por ende, el número de estudios primarios candidatos para ser meta-analizables sea reducido, lo que supone que la aplicación del metaanálisis carecería de potencia estadística y de capacidad de generalización.

Otra limitación relevante tiene que ver con el tipo de diseño empleado en los estudios primarios, dado que la gran mayoría son ex post facto (empleando en su mayoría análisis de correlaciones, donde no se puede analizar direccionalidad ni causalidad). En este tipo de diseños, no se puede establecer si altos niveles de fortalezas se relacionan con un mayor bienestar, o si son estos altos niveles de bienestar los que potencian la manifestación de estos rasgos positivos.

Vistos los beneficios asociados a la identificación y puesta en marcha de las fortalezas y el balance de las limitaciones del presente estudio, se hace necesario señalar una serie de implicaciones y perspectivas para futuros estudios. En el área individual, se destaca la importancia de que cada persona conozca cuáles son sus fortalezas y cómo ponerlas en marcha en diferentes contextos. Identificar y ser capaz de desarrollarlas puede producir aumento en el bienestar como se muestra en el estudio de Proyer Ruch y Buschor (2012) y en los meta-análisis realizados por Bolier et al. (2013) y Sin y Lyubomirsky (2009), además de contribuir a reducir los síntomas depresivos (Proyer, Gander, Wellenzohn y Ruch, 2016). Se ha mostrado también que las fortalezas generan recursos que pueden fomentar la resistencia ante la adversidad e incluso un afrontamiento adaptativo ante la enfermedad física (Peterson et al., 2006). Estos resultados destacan la importancia de desarrollar las fortalezas psicológicas en adultos, y además subrayan el rol decisivo de programas educativos en la infancia y adolescencia, dado que son momentos evolutivos clave para establecer y fomentar aspectos positivos en el ser humano (Ferragut, Blanca, y OrtizTallo, 2014). Park y Peterson (2003a; 2003b; 2006) apuntan que las fortalezas en los jóvenes son una clave fundamental para el bienestar de la sociedad en el futuro. Diferentes estudios han mostrado el papel de las diferentes fortalezas en el bienestar y la satisfacción vital en la infancia y la adolescencia (Blanca, Ferragut, Ortiz-Tallo y Bendayan, en prensa; Park y Peterson, 2006; 2008; Ruch, Weber, Park, y Peterson, 2014) e incluso a la hora de prevenir actitudes sexistas (Ferragut, Blanca y OrtizTallo, 2013) y potenciar la autoestima y la autoeficacia en niños hospitalizados (Toback, Graham-Bermann, y Patel, 2016), y el bienestar en niños de altas capacidades (Pfeiffer, 2016). En personas mayores, también se han visto las importantes contribuciones de las fortalezas como factores que fomentan un envejecimiento más saludable (Ramírez et al., 2015).

Desde una perspectiva social y comunitaria, el desarrollo de las fortalezas puede contribuir a la promoción de la salud, encajando así la Psicología Positiva y dentro de ella, las fortalezas psicológicas, como una disciplina que se esfuerza en promover el desarrollo los aspectos positivos del ser humano en los individuos, en los grupos y en la sociedad en general (Guðrún Guðmundsdóttir, 2015). En este sentido, la incorporación del vocabulario sobre fortalezas en el día a día contribuiría a cambiar la perspectiva sobre el concepto de salud mental, partiendo de un enfoque de fomento de la resiliencia social (Ovejero, 2015; Seligman, 2015).

\section{Referencias}

*Artículos incluidos en la revisión sistemática

1. *Allan, B. A., y Duffy, R. D. (2014). Examining moderators of signature strengths use and well-being: Calling and signature strengths level. Journal of Happiness Studies, 15, 323-337. https://doi.org/10.1007/s10902-013-9424-0

2. American Psychiatric Association. (2013). Diagnostic and statistical manual of mental disorders (5a Ed.). Arlington, VA: American Psychiatric Publishing. https://doi. org/10.1176/appi.books.9780890425596

3. *Azañedo, C. M., Fernández-Abascal, E. G., y Barraca, J. (2014). Character strengths in Spain: Validation of the Values in Action Inventory of Strengths (VIA-IS) in a Spanish sample. Clínica y Salud, 25, 123-130. https://doi. org/10.1016/j.clysa.2014.06.002

4. *Berman, J. S. (2007). Character strengths, self-schemas, and psychological well being: A multi-method approach. Tesis doctoral. Universidad de Texas. Disponible en: https://repositories.tdl.org/utswmed-ir/bitstream/handle/ 2152.5/507/BermanJason.pdf? sequence $=6$ 
5. Blanca, M., Ferragut, M., Ortiz-Tallo, M., y Bendayan, R. (en prensa). Life satisfaction and character strengths in Spanish early adolescents. Journal of Happiness Studies.

6. Bolier, L., Haverman, M., Westerhof, G. J., Riper, H., Smit, F., y Bohlmeijer, E. (2013). Positive psychology interventions: A meta-analysis of randomized controlled studies. BMC Public Health, 13, 119-139. https://doi. org/10.1186/1471-2458-13-119.

7. *Brdar, I. Anić, P., y Rijavec, M. (2011). Character strengths and well-being: are there gender differences? En I. Brdar (Ed.), The Human Pursuit of Well-being (pp.145156). Nueva York: Springer. https://doi.org/10.1007/97894-007-1375-8 13

8. *Brdar, I., y Kashdan, T. B. (2010). Character strengths and well-being in Croatia: An empirical investigation of structure and correlates. Journal of Research in Personality, 44, 151-154. https://doi.org/10.1016/j.jrp.2009.12.001

9. *Buschor, C., Proyer, R. T., y Ruch, W. (2013). Self- and peer-rated character strengths: How do they relate to satisfaction with life and orientations to happiness? The Journal of Positive Psychology, 8, 116-127. https://doi.org/10.108 0/17439760.2012.758305.

10. *Dahlsgaard, K. K. (2005). Is virtue more than its own reward? Character strengths and their relation to well-being in a prospective longitudinal study of middle school-aged adolescents. Tesis Doctoral. Universidad de Pensilvania http://repository.upenn.edu/dissertations/AAI3179723

11. Dahlsgaard, K., Peterson, C., y Seligman, M. E. (2005). Shared virtue: The convergence of valued human strengths across culture and history. Review of General Psychology, 9, 203-213. https://doi.org/10.1037/1089-2680.9.3.203

12. Ferragut, M., Blanca, M. J., y Ortiz-Tallo, M. (2013). Psychological values as protective factors against sexist attitudes in preadolescents. Psicothema, 25, 38-42. https://doi. org/10.7334/psicothema2012.85

13. Ferragut, M., Blanca, M. J., y Ortiz-Tallo, M. (2014). Psychological virtues during adolescence: a longitudinal study of gender differences. European Journal of Developmental Psychology, 11, 521-531. https://doi.org/10.1080/1740562 9.2013.876403

14. *Forest, J., Mageau, G. A., Crevier-Braud, L., Bergeron, É., Dubreuil, P., y Lavigne, G. L. (2012). Harmonious passion as an explanation of the relation between signature strengths' use and well-being at work: Test of an intervention program. Human Relations, 65, 1233-1252. https:// doi.org/10.1177/0018726711433134

15. *Gander, F., Proyer, R. T., Wyss, T., y Ruch, W. (2012). The good character at work: an initial study on the contribution of character strengths in identifying healthy and unhealthy work-related behavior and experience patterns. International Archives of Occupational and Environmental Health, 85, 1-10. doi. https://doi.org/10.1007/s00420-012-0736-x

16. *Gander, F., Proyer, R. T., Ruch, W., y Wyss, T. (2012).
Strength-based positive interventions: Further evidence for their potential in enhancing well-being and alleviating depression. Journal of Happiness Studies, 14, 1241-1259. https://doi.org/10.1007/s10902-012-9380-0.

17. *Gillham, J., Adams-Deutsch, Z., Werner, J., Reivich, K., Coulter-Heindl, V., Linkins, M., ..., y Contero, A. (2011). Character strengths predict subjective well-being during adolescence. The Journal of Positive Psychology, 6, 31-44. https://doi.org/10.1080/17439760.2010.536773

18. *Giménez, M. (2010). La medida de las fortalezas psicológicas en adolescentes (VIA-Youth): Relación con clima familiar, psicopatología y bienestar psicológico. Tesis doctoral. Universidad Complutense de Madrid. Disponible en: http://eprints.ucm.es/11578/1/T32253.pdf

19. Goodman, F. R., Disabato, D. J., Kashdan, T. B., y Machell, K. A. (2016). Personality strengths as resilience: A oneyear multiwave study. Journal of Personality. https://doi. org/10.1111/jopy.12250.

20. Govindji, R., y Linley, P. A. (2007). Strengths use, self-concordance and well being: Implications for strengths coaching and coaching psychologists. International Coaching Psychology Review, 2, 143-153.

21. *Gradisek, P. (2012). Character strengths and life satisfaction of Slovenian in-service and pre-service teachers. CEPS Journal: Center for Educational Policy Studies Journal, 2, 167-180.

22. *Grinhauz, A. S. (2015). El estudio de las fortalezas del carácter en niños: relaciones con el bienestar psicológico, la deseabilidad social y la personalidad. Psicodebate, 15, 43-68. https://doi.org/10.18682/pd.v15i1.483

23. Guðrún Guðmundsdóttir, D. (2015). Positive psychology and public health. En R. Biswas-Diener (Ed.), Positive Psychology as Social Change (pp 109-124). Nueva York: Springer.

24. *Güsewell, A., y Ruch, W. (2012). Are only emotional strengths emotional? Character strengths and disposition to positive emotions. Applied Psychology: Health and Well-being, 4, 218-239. https://doi.org/10.1111/j.1758$\underline{0854.2012 .01070 . \mathrm{x}}$

25. *Gustems, J., y Calderon, C. (2014). Character Strengths and Psychological Well-being among Students of Teacher Education. International Journal of Educational Psychology, 3, 265-286. https://doi.org/10.1007/s10212-012-0158-x

26. *Harzer, C., y Ruch, W. (2013). The application of signature character strengths and positive experiences at work. Journal of Happiness Studies, 14, 965-983. https:// doi.org/10.1007/s10902-012-9364-0

27. *Hool, K. (2011). Character strengths, life satisfaction and orientations to happiness-a study of the Nordic countries. Tesis doctoral. Universidad de Bergen. Disponible en: http://bora.uib.no/bitstream/handle/1956/5758/91892545. pdf? sequence $=1$ \&isAllowed $=y$

28. *Imura, T., Aoki, T., Takahashi, T., Nonaka, Y., y Ya- 
mada, T. (2013). Character strengths and well-being in Japanese children and youth: Creating good habits. The Japanese Journal of Psychology, 84, 247-255. https://doi. org/10.4992/jijpsy.84.247.

29. Institute of Medicine of the National Academies (2011). Finding what works in health care: Standards for initiating a Systematic Review. Washington DC: Institute of Medicine of the National Academies. Disponible en: http://www. nationalacademies.org/hmd/Reports/2011/Finding-WhatWorks-in-Health-Care-Standards-for-Systematic-Reviews/ Standards.aspx

30. *Karris, M. A. (2007). Character strengths and well-being in a college sample. Tesis doctoral. Universidad de Colorado.

31. *Khumalo, I. P., Wissing, M. P., y Temane, Q. M. (2008). Exploring the validity of the Values-In-Action Inventory of Strengths (VIA-IS) in an African context. Journal of Psychology in Africa, 18, 133-142.

32. *Lavy, S., y Littman-Ovadia, H. (2011). All you need is love? Strengths mediate the negative associations between attachment orientations and life satisfaction. Personality and Individual Differences, 50, 1050-1055. https://doi. org/10.1037/e537902012-011

33. *Lee, J. N. T., Foo, K. H., Adams, A., Morgan, R., y Frewen, A. (2015). Strengths of character, orientations to happiness, life satisfaction and purpose in Singapore. Journal of Tropical Psychology, 5. https://doi.org/10.1017/jtp.2015.2

34. *Linley, P. A., Nielsen, K. M., Gillett, R., y Biswas-Diener, R. (2010). Using signature strengths in pursuit of goals: Effects on goal progress, need satisfaction, and well-being, and implications for coaching psychologists. International Coaching Psychology Review, 5, 6-15.

35. Littman-Ovadia, H., y Davidovitch, N. (2010). Effects of congruence and character-strength development on work adjustment and well-being. International Journal of Business and Social Science, 1, 138-146.

36. *Littman-Ovadia, H., y Lavy, S. (2012). Differential ratings and associations with well-being of character strengths in two communities. Health Sociology Review, 21, 299-312. https://doi.org/10.5172/hesr.2012.1378

37. Littman-Ovadia, H., y Steger, M. (2010). Character strengths and well-being among volunteers and employees: Toward an integrative model. Journal of Positive Psychology, 5, 419-430. https://doi.org/10.1080/17439760.2010.5 $\underline{16765}$

38. *Lounsbury, J. W., Fisher, L. A., Levy, J. J., y Welsh, D. P. (2009). An investigation of character strengths in relation to the academic success of college students. Individual Differences Research, 7, 52-69.

39. Macdonald, C., Bore, M., y Munro, D. (2008). Values in action scale and the big 5: An empirical indication of structure. Journal of Research in Personality, 42, 787-799. https://doi.org/10.1016/j.jrp.2007.10.003
40. *Martínez-Martí, M. L., y Ruch, W. (2016). Character strengths predict resilience over and above positive affect, self-efficacy, optimism, social support, self-esteem, and life satisfaction. The Journal of Positive Psychology. https:// doi.org/10.1080/17439760.2016.1163403

41. McGrath, R. (2014). Scale-and item-level factor analyses of the VIA inventory of strengths. Assessment, 21, 4-14. https://doi.org/10.1177/1073191112450612

42. McGrath, R. (2015). Measuring invariance in translations of the VIA Inventory of Strengths. European Journal of Psychological Assessment, 32, 187-194. https://doi. org/10.1027/1015-5759/a000248

43. *Moradi, S., Nima, A. A., Rapp Ricciardi, M., Archer, T., y Garcia, D. (2014). Exercise, character strengths, well-being, and learning climate in the prediction of performance over a 6-month period at a call center. Frontiers in psychology, 5. https://doi.org/10.3389/fpsyg.2014.00497

44. Moher, D., Liberati, A., Tetzlaff, J., Altman, D. G., y The PRISMA Group (2009). Preferred Reporting Items for Systematic Reviews and MetaAnalyses: The PRISMA Statement. PLoS Medicine, 6: e1000097. https//doi.org/10.1371/ journal.pmed1000097

45. Niemiec, R. M. (2010). Then principles of character strengths. Positive Psychology News Daily. Disponible en: http://positivepsychologynews.com/news/ryan-niemiec /2010052611161

46. Niemiec, R. M. (2013). VIA character strengths: research and practice (the first 10 years). Cross-cultural Advancements in Positive Psychology, 3, 11-29. https://doi. org/10.1007/978-94-007-4611-4_2

47. Noftle, E. E., Schnitker, S. A., y Robins, R. W. (2011). Character and personality: Connections between Positive Psychology and Personality Psychology. En K.M. Sheldon, T. B. Kashdan y M. F. Steger (Eds.), Designing Positive Psychology. Taking Stock and Moving Forward (pp 207227). Nueva York: Oxford University Press.

48. *Ovejero, M. (2014). Evaluación de fortalezas humanas en estudiantes de la Universidad Complutense de Madrid $y$ diferencias de sexo: Relación con salud, resiliencia $y$ rendimiento académico. Tesis doctoral. Universidad Complutense de Madrid.

49. Ovejero, M. (2015). Las fortalezas como arquetipo de la humanidad. Revista Mexicana de Investigación en Psicología, 7, 118-124.

50. Ovejero, M., y Cardenal, V. (2015). Las fortalezas humanas desde la perspectiva de género: un estudio exploratorio en población española. Revista Mexicana de Investigación en Psicología, 7, 72-92.

51. Park, N., y Peterson, C. (2003a). Moral competence and character strengths among adolescents: The development and validation of the Values in Action Inventory of Strengths for youth. Journal of Adolescence, 29, 891-910. https://doi.org/10.1016/j.adolescence.2006.04.011 
52. Park, N., y Peterson, C. (2003b). Assessment of character strengths among youth: The Values In Action Inventory of Strengths for youth. Conferencia presentada en la Positive Development Conference. Washington, 12-13 de Marzo de 2003.

53. Park, N., y Peterson, C. (2006). Character strengths and happiness among young children: context analysis of parental descriptions. Journal of Happiness Studies, 7, 323341. https://doi.org/10.1007/s10902-005-3648-6

54. *Park, N., Peterson, C., y Seligman M. E. P. (2004a). Strengths of character and well-being. Journal of Social and Clinical Psychology, 23, 603-619. https://doi.org/10.1521/ jscp.23.5.603.50748

55. Park, N., Peterson, C., y Seligman M. E. P. (2004b). Reply: strengths of character and well-being: a closer look at hope and modesty. Journal of Social and Clinical Psychology, 23, 628-634. https://doi.org/10.1521/jscp.23.5.628.50749

56. Peterson, C. (2006a). The Values In Action (VIA) classification of strengths. En M. Czikszentmihalyi, y I. S. Czikszentmihalyi (Eds.), A Live Worth Living. Contributions to Positive Psychology (pp 29-48). Nueva York. Oxford University Press.

57. Peterson, C. (2006b). Strengths of character and happiness: Introduction to special issue. Journal of Happiness Studies, 7, 289-291. https://doi.org/10.1007/s10902-005-3645-9

58. Peterson, C., y Park, N. (2009). El estudio científico de las fortalezas humanas. En C. Vázquez y G. Hervás (coords.), La Ciencia del Bienestar. Fundamentos de una Psicología Positiva (pp. 181-207). Madrid. Alianza Editorial.

59. Peterson, C., y Park, N. (2011). Character strengths and virtues: Their role in well-being. En S.I. Donaldson, M. Csikszentmihaly, y J. Nakamura (Eds.), Applied Positive Psychology. Improving Everyday Life, Health, Schools, Work and Society (pp. 49-62). Nueva York: Routledge.

60. Peterson, C., y Park, N. (2013). Character strengths and the life of meaning. En P. Wong (Ed.), The Human Quest for Meaning: Theories, Research, and Applications (pp. 277294). Nueva York: Routledge.

61. *Peterson, C., Park, N., Pole, N., D’Andrea, W., y Seligman, M. E. (2008). Strengths of character and posttraumatic growth. Journal of Traumatic Stress, 21, 214-217. https://doi.org/10.1002/jts.20332

62. Peterson, C., Park, N., y Seligman, M. E. P. (2005). Assessment of character strengths. En G. P. Koocher, J. C. Norcross, y S. S. Hill III (Eds.), Psychologists'desk reference (pp. 93-98). Nueva York, NY: Oxford University Press.

63. Peterson, C., Park, N., y Seligman, M. E. P. (2006). Greater strengths of character and recovery from illness. Journal of Positive Psychology, 1, 17-26. https://doi. org/10.1080/17439760500372739

64. *Peterson, C., Ruch, W., Beermann, U., Park, N., y Seligman, M. E. P. (2007). Strengths of character, orientations to happiness, and life satisfaction. Journal of Positive Psychology,
2, 149-156. https://doi.org/10.1080/17439760701228938

65. Peterson, C. y Seligman, M. E. P. (2004). Character strengths and virtues. A handbook and classification. Nueva York: Oxford University Press.

66. Peterson, C., Stephens, J. P., Park, N., Lee, F., y Seligman, M. E. P. (2010). Strengths of character and work. En A. Linley, S. Harrington y N. Garcea (Eds.), Oxford Handbook of Positive Psychology and Work (pp 221-231). Nueva York: Oxford Library of Psychology.

67. Pfeiffer, S. I. (2016). Success in the classroom and in life. Focusing on strengths of the head and strengths of the heart. Gifted Education International, 1-7 https://doi. org/10.1177/0261429416640337.

68. Proctor, C. (2013). The importance of good character. En C. Proctor, y A. Linley (Eds.), Research, Applications, and Interventions for Children and Adolescents (pp. 13-21). Nueva York: Springer. https://doi.org/10.1007/978-94-0076398-2 2

69. *Proctor, C., Maltby, J., y Linley, P. A. (2011). Strengths use as a predictor of well-being and health-related quality of life. Journal of Happiness Studies, 12, 153-169. https:// doi.org/10.1007/s10902-009-9181-2

70. *Proyer, R. T., Gander, F., Wellenzohn, S., y Ruch, W. (2013). What good are character strengths beyond subjective well-being? The contribution of the good character on self-reported health-oriented behavior, physical fitness, and the subjective health status. Journal of Positive Psychology, 8, 222-232. https://doi.org/10.1080/17439760.2013.7 77767.

71. Proyer, R. T., Gander, F., Wellenzohn, S., y Ruch, W. (2016). Nine beautiful things: A self-administered online positive psychology intervention on the beauty in nature, arts and behaviors increases happiness and ameliorated depressive symptoms. Personality and Individual Differences, 94, 189-193. https://doi.org/10.1016/j.paid.2016.01.028

72. *Proyer, R. T., Gander, F., Wyss, T., y Ruch, W. (2011). The relation of character strengths to past, present, and future life satisfaction among German-speaking women. Applied Psychology: Health and Well-being, 3, 370-384. https:// doi.org/10.1111/j.1758-0854.2011.01060.x

73. Proyer, R. T., Ruch, W., y Buschor, C. (2012). Testing strengths-based interventions: a preliminary study on the effectiveness of a program targeting curiosity, gratitude, hope, humor and zest for enhancing life satisfaction. Journal of Happiness Studies, 13. $\quad$ https://doi.org/10.1007/ s10902-012-9331-9

74. *Ramirez, E., Ortega A. R., y Martos, R. (2015). Las fortalezas en personas mayores como factor que aumenta el bienestar. European Journal of Investigation in Health, Psychology and Education, 5, 187-195. https//doi. org/10.1989/ejihpe.v5i2.111.

75. *Ruch, W., Proyer, R. T., Harzer, C., Park, N., Peterson, C., y Seligman, M. E. (2010). Values in action inventory of 
strengths (VIA-IS): adaptation and validation of the German version and the development of a peer-rating form. Journal of Individual Differences, 31, 138. https://doi. org/10.1037/t03517-000

76. *Ruch, W., Weber, M., Park, N. y Peterson C., (2014). Character strengths in children and adolescents. Reliability and initial validitiy of the German Values in Action Inventory of Strengths for Youth (German VIA-Youth). European Journal of Psychological Assessment, 30, 57-64. https:// doi.org/10.1027/1015-5759/a000169

77. Seligman, M. E. P. (2015). Chris Peterson's unfinished masterwork: The real mental illnesses. The Journal of Positive Psychology, 10, 3-6. https://doi.org/10.1080/1743976 $\underline{0.2014 .888582}$

78. Seligman, M. E. P., y Csikszentmihalyi, M. (2000). Positive Psychology. An introduction. American Psychologist, 55, 5-14. https://doi.org/10.1037//0003-066X.55.1.5

79. Seligman, M. E. P., Steen, T. A., Park, N., y Peterson, C. (2005). Positive psychology progress. Empirical validation of interventions. American Psychologist. 60, 410-421. https://doi.org/10.1037/0003-066X.60.5.410

80. *Shimai, S., Otake, K., Park, N., Peterson, C., y Seligman, M. E. (2006). Convergence of character strengths in American and Japanese young adults. Journal of Happiness Studies, 7, 311-322. https://doi.org/10.1007/s10902-005-36477

81. *Shoshani, A., y Slone, M. (2013). Middle school transition from the strengths perspective: Young adolescents' character strengths, subjective well-being, and school adjustment. Journal of Happiness Studies, 14(4), 1163-1181. https://doi.org/10.1007/s10902-012-9374-y

82. *Shoshani, A., y Slone, M. (2015). The resilience function of character strengths in the face of war and protracted conflict. Frontiers in Psychology. https://doi. org/10.3389\%2Ffpsyg.2015.02006

83. Sin, N. L., y Lyubomirsky, S. (2009). Enhancing well-being and alleviating depressive symptoms with positive psychology interventions: a practice-friendly meta-analysis. Journal of Clinical Psychology: In Session, 65, 467-487. https://doi.org/10.1002/jclp.20593.
84. Toback, R. L., Graham-Bermann, S. A., y Patel, P. D. (2016). Outcomes of a character strengths-based intervention on self-esteem and self-efficacy of psychiatrically hospitalized youths. Psychiatric Services, 67, 574-577. https:// doi.org/10.1176/appi.ps.201500021

85. *Toner, E., Haslam, N., Robinson, J., y Williams, P. (2012). Character strengths and wellbeing in adolescence: Structure and correlates of the Values in Action Inventory of Strengths for Children. Personality and Individual Differences, 52, 637-642. https://doi.org/10.1016/j.paid.2011.12.014

86. *Weber, M., y Ruch, W. (2012). The role of a good character in 12-year-old school children: Do character strengths matter in the classroom? Child Indicators Research, 5, 317334. https://doi.org/10.1007/s12187-011-9128-0

87. *Weber, M., y Ruch, W. (2012). The role of character strengths in adolescent romantic relationships: An initial study on partner selection and mates' life satisfaction. Journal of adolescence, 35, 1537-1546. https://doi. org/10.1016/j.adolescence.2012.06.002

88. *Weber, M., Ruch, W., Littman-Ovadia, H., Lavy, S., y Gai, O. (2013). Relationships among higher-order strengths factors, subjective well-being, and general self-efficacy-The case of Israeli adolescents. Personality and Individual Differences, 55, 322-327. https://doi.org/10.1016/j. paid.2013.03.006

89. Wood, A. M, Linley, A., Maltby, J., Kashdan, T. B., y Hurling, R. (2011). Using personal and psychological strengths leads to increases in well-being over time: A longitudinal study and the development of the strengths use questionnaire. Personality and Individual Differences, 50, 15-19. https://doi.org/10.1016/j.paid.2010.08.004

90. *Zhou, Y., Siu, A. F. Y., y Liu, X. P. (2013). What does not kill me makes me stronger: The relationship between depression history, character strengths and coping strategies. Indian Journal of Health and Wellbeing, 4, 1249-1254.

RECIBIDO: $14 / 06 / 2016$ MODIFICADO: 09/11/2016 ACEPTADO:23/11/2016 